\title{
Impact and Future Changes to Breast Cancer Care Due to Covid-19
}

\author{
Kennard Kaitlyn* and Carp Ned \\ Lankenau Institute for Medical Research, USA \\ *Corresponding author: Kennard Kaitlyn, Lankenau Institute for Medical Research, USA. \\ To Cite This Article: Kennard Kaitlyn, Carp Ned. Impact and Future Changes to Breast Cancer Care Due to Covid-19. Am J Biomed Sci \& Res. \\ 2021 - 14(3). AJBSR.MS.ID.001987. DOI: 10.34297/AJBSR.2021.14.001987.
}

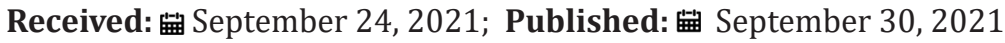

\begin{abstract}
Abbreviations: WHO: World Health Organization; SARS-CoV2: Severe Acute Respiratory Syndrome Coronavirus 2; COVID-19: Coronavirus Disease 2019; NCCN: National Comprehensive Cancer Network; ACR: American College of Radiology; ASBrS: American Society of Breast Surgeons; NCT: Neoadjuvant Chemotherapy; CoC: Commission on Cancer
\end{abstract}

\section{Introduction}

A novel coronavirus severe acute respiratory syndrome coronavirus 2 (SARS-CoV2) was identified and the World Health Organization (WHO) termed with illness it caused coronavirus disease 2019 (COVID-19). This occurred in late 2019 and was first identified in Wuhan City, Hubei Province, China [1]. By the beginning of March 2020 many restrictions were in place across the United States to decrease transmission of the virus and the disease burden on health systems. These restrictions to contain COVID-19 impacted various aspects of the health systems across the nation and their ability to deliver care. Specifically, the ability of health systems to respond to emergencies, obtain sufficient health care supplies and maintain adequate numbers of health staff were compromised [2,3]. Early in the pandemic, several large United States (US) based medical societies including the American Society of Breast Surgeons (ASBrS), the National Accreditation Program for Breast Centers (NAPBC), the National Comprehensive Cancer Network (NCCN), the Commission on Cancer (CoC) and the American College of Radiology (ACR) collaborated to provide expert opinion regarding how to prioritize patients diagnosed with breast cancer during this time [4]. These recommendations placed patients into different groups based on the acuity of disease and risk of disease progression while evaluating the risk of intervention based on hospital resources and risk of personnel exposure. One main recommendation from this risk stratification schema was the prioritization of surgical intervention for patients completing neoadjuvant chemotherapy (NCT) for triple negative and HER2positive disease. In addition, it was recommended to initiate neoadjuvant endocrine therapy (NET) for patients with early-stage hormone positive breast cancer [46]. Local and national databases were created to evaluate the impact of these expert opinion guidelines on the management of breast cancer in the US. The ASBrS leadership established a Mastery of Breast Surgery database March 2020 to capture these changes in breast cancer care during the COVID-19 pandemic for which the first 12 months of outcomes were just published. All surgeons who entered into the database reported that they had some interruption in their elective surgical schedule due to COVID-19. Surgeons in the registry were across the US with $36.7 \%$ being the in northeastern US. Mean patient age was 62 years old and 11\% were African American. Telehealth was used for $6.2 \%$ of patients and $1.4 \%$ developed COVID-19. The mean invasive tumor size was $2.1 \mathrm{~cm}$ with $17.8 \%$ of patients being node-positive and $10.2 \%$ were triple negative [4-6]. In the Mastery registry the mean time to primary surgery was 44.5 days. NET was used in additional $24.3 \%$ of patients with hormone positive breast cancer and $30.8 \%$ of patient with DCIS. An increased percentage of patients in the northeast received NET. Genomic testing on the core biopsy occurred more frequently due to COVID-19 in patients who were node-positive. The surgeons reported that $12.4 \%$ of patients were return for another surgery later, and the majority of these surgeries were for mastectomy with or without reconstruction. For 
this national database a change in surgical approach was reported for $5.4 \%$ of patients with $11.5 \%$ of patients still having surgery pending at time of analysis [4-6]. In this national registry we see that breast cancer care was altered with increased use of NET and genomic testing on core biopsy.

The impact on cancer and non-cancer health outcomes may take years to fully understand. Another study captured changes across a health system in the northeast which was an epicenter of COVID-19 [7]. In this analysis $44 \%$ of patients experienced a change in treatment with $65.6 \%$ of patients with a change in treatment receiving NET and 34.4\% experiencing a delay in surgery alone. For those who had a delay in surgery date due to COVID-19 the median time to surgery was 70.5 days but those receiving NET has a median time to surgery of 85 days. Patients were also given a survey Generalized Anxiety Disorder two-item (GAD-2) questionnaire which revealed that $31.2 \%$ of patients screened positive for anxiety and depression. Interestingly this was not statistically different between women who had a change in care due to COVID-19 or rates of GAD-2 positivity for women with breast cancer outside of the COVID-19 pandemic [7-8]. From multiple studies on both the local and national level, COVID-19 caused delays in breast cancer care through the guidance of national organizations and local mandates. The changes in breast cancer care did vary by location due to burden of disease and local government responses. Across the US the major changes in care seen due to the pandemic were use of NET and delay in surgical dates. The pandemic continues to affect many areas geographically today and those impacted by the current wave of COVID-19 is largely different than the first wave. From these studies we see that there were changes implemented in the care of breast cancer patients. Fortunately, women were very resilient and despite alterations in their breast cancer care there was no increase in levels of anxiety and depression. Moving forward this speaks to the importance of the breast cancer team in communicating with the patient. Although cancer care my change patients are ready to embrace this $[4,6-8]$.
One change implemented here in the US was increased use of neoadjuvant endocrine therapy for hormone positive breast cancer. This was implemented during the COVID pandemic due to difficulty in enabling many breast cancer patients to proceed to the operating room with shortages of staff and personal protective equipment [4,6-8]. Many assessments are underway to evaluate breast cancer outcomes for this cohort. Alterations in breast cancer care due to COVID-19 were rapid this will likely open up many possibilities for future directions of breast cancer care.

\section{References}

1. (2020) WHO Timeline - COVID-19.

2. Habibzadeh P, Stoneman EK (2020) The Novel Coronavirus: A Bird's Eye View. Int J Occup Environ Med 11(2): 65-71.

3. Jin X, Lian JS, Hu JH, Jianguo Gao, Lin Zheng, et al. (2020) Epidemiological, clinical virological characteristics of 74 cases of coronavirus-infected disease 2019 (COVID-19) with gastrointestinal symptoms. Gut 69(6):1002-1009.

4. Jill R Dietz, Meena S Moran, Steven J Isakoff, Scott H Kurtzman, Shawna C Willey, et al. (2020) Recommendations for prioritization, treatment, and triage of breast cancer patients during the COVID-19 pandemic. the COVID-19 pandemic breast cancer consortium. Breast Cancer Res Treat 181(3): 487-497.

5. Jennifer Y Sheng, Cesar A SantaMaria, Neha Mangini, Haval Norman, Rima Couzi, et al. (2020) Resource for Management Options of Breast Cancer During COVID-19. Oncology SoS 16(10): 665-674.

6. Wilke LG, Nguyen TT, Yang Q Bret M Hanlon, Kathryn A Wagner, et al. (2021) Analysis of the Impact of the COVID-19 Pandemic on the Multidisciplinary Management of Breast Cancer: Review from the American Society of Breast Surgeons COVID-19 and Mastery Registries. Ann Surg Oncol 16(1)

7. Kennard K, Williams A, Goldblatt L, Meghan E Buckley, Laura Bruce, et al. ( 2021) COVID-19Pandemic: Changes in Care for a Community Academic Breast Center and PatientPerception of those Changes. Annals of Surgical Oncology 28(9): 5071-5081.

8. Kennard K, Sizer LM, Frazier TG (2021) ASO Author Reflections: Changes in Breast Cancer Care and Patient Perception During the Initial Wave of COVID-19. Ann Surg Oncol 28(9): 5082-5083. 